Supporting Information

\title{
Self-Synchronous Swinging Motion of a Pair of
}

\section{Autonomous Droplets}

Shiho Sato ${ }^{\dagger}$, Hiroki Sakuta ${ }^{\dagger}$, Koichiro Sadakane, Kenichi Yoshikawa*

Faculty of Life and Medical Sciences, Doshisha University, Kyoto 610-0394, Japan

${ }^{\dagger}$ Contributed equally

Corresponding Author

*keyoshik@mail.doshisha.ac.jp; Phone: +81-774-65-6243 


\section{Supplement S1}

To reveal the character of the synchronization, we observed the 1:1 swinging-type synchronization, in the case of giving a disturbance on the synchronized state. Figure S1 (a) shows the time dependent tracing of two droplets, initially the two droplets exhibit the synchronization. At the $T=10 \mathrm{sec}$, the left side droplet was disturbed its motion by dipping another glass rod to the left waterway and the synchronization of two droplets was once broken, as the part of the broken line of Fig. S1 (b). After several ten seconds, the 1:1 swinging-type synchronization recovered spontaneously.

(a)

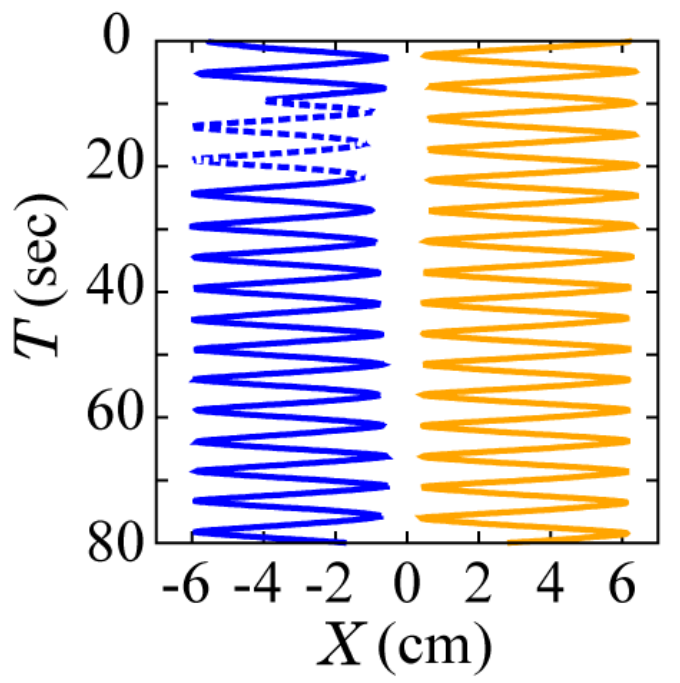

(b)

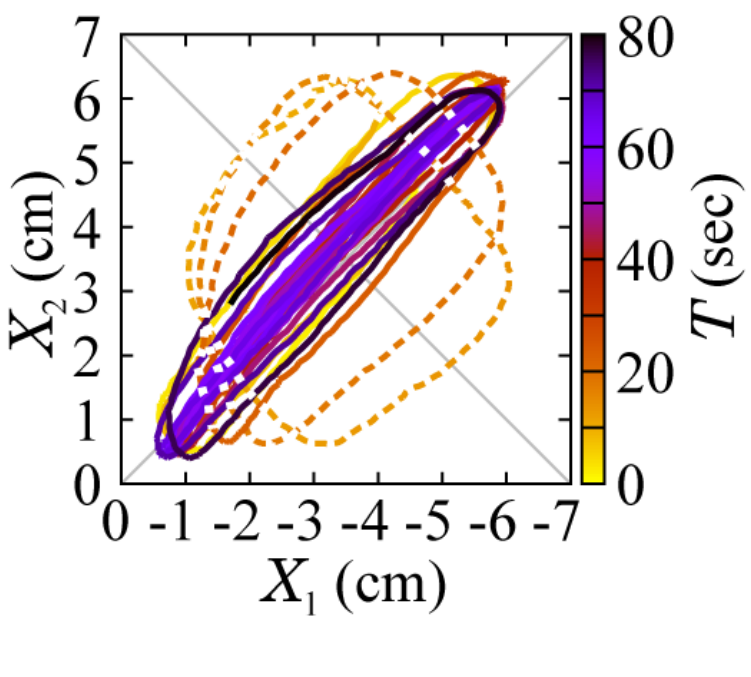

Figure S1. Experimental observation on the effect of a disturbance on the synchronized state, as depicted by a (a) spatio-temporal diagram and (b) correlation. A glass rod was placed on the left side of the left waterway under the same condition as in Fig. 2 (a1), for a period of $1 \mathrm{sec}$ from the timing of $T=10 \mathrm{sec}$. The broken line indicates the motion of the left droplet just after the disturbance. The 1:1 synchronization spontaneously recovered after ca. $10 \mathrm{sec}$. 